\title{
Medidas antropométricas e nível de atividade física predizem pressão arterial elevada em crianças
}

\author{
Anthropometric measurements and physical activity level predict high \\ blood pressure in children
}

Alynne C. R. Andaki1',2, Edmar L. Mendes², Wellington Segheto', Fernanda S. Franco', Adelson L. A. Tinôco

\section{Resumo}

O presente estudo teve como objetivo verificar a acurácia de medidas antropométricas e do nível de atividade física (NAF) como preditores do risco de pressão arterial (PA) elevada, por sexo, em crianças. Participaram do estudo 187 crianças, média de idade de 9,9 \pm 0,7 anos, de ambos os sexos, estudantes de nove escolas (públicas e privadas) do município de Viçosa-MG em 2009. As crianças foram submetidos a avaliação antropométrica, composição corporal e aferição da PA. Em seguida, preencheram registro de atividades físicas e utilizaram pedômetro por sete dias. Foram delineadas curvas Receiver Operating Characteristic (ROC) para testar a acurácia das medidas antropométricas e NAF em predizer o risco de PA elevada. Índice de Massa Corporal (IMC), perímetros de cintura mensurados em três pontos anatômicos, somatório de quatro dobras cutâneas e NAF foram acurados em predizer PA elevada para as meninas. Por outro lado, para os meninos apenas o número de passos/dia foi acurado na predição do PA elevada. NAF prediz PA elevada em crianças, independente do sexo, enquanto medidas antropométricas prediz PA elevada apenas para crianças do sexo feminino.

\section{Palavras-chave}

Hipertensão; Criança; Atividade motora; Antropometria.

\begin{abstract}
This study aimed to verify the accuracy of anthropometric measurements and the physical activity level (PAL) as predictors of the risk of high blood pressure $(H B P)$ in children. Participated in the study 187 children of both sexes, aged $9.9 \pm 0.7$ years, from nine public and private schools in the city of Viçosa-MG in 2009. Children underwent an anthropometric measurements, body composition and blood pressure measurement. The children met record physical activities and used pedometer for seven days. Receiver operating characteristic (ROC) curves were outlined to test the accuracy of anthropometric measurements and PAL in predicting the risk of HBP. Body Mass Index (BMI), waist circumference measured at three sites, sum of four skinfolds, and PAL were accurate in predicting risk of HBP for girls. On the other hand, for boys only the number of steps/day was accurate in predicting HBP. Physical activity level in children predicts HBP irrespective of sex while anthropometric measurements predict HBP only for girls.
\end{abstract}

\section{Keywords}

Hypertension; Child; Motor Activity; Anthropometry.

\section{Introdução}

A prevalência de hipertensão arterial sistólica (HAS) em crianças tem aumentado consideravelmente nos últimos anos ${ }^{1}$. No Brasil, a Sociedade Brasileira de Hipertensão estimou em 3,5 milhões o número de crianças hipertensas com necessidade de tratamento $^{2}$. Estudos conduzidos no Brasil demonstraram prevalência de HAS entre 2,5 a 30,9\% e alertaram que a ampla variação se deve aos distintos procedimentos metodológicos utilizados nos estudos ${ }^{3}$.

1 Programa de Pós-graduação em Ciência da Nutrição Universidade Federal de Viçosa (UFV), Viçosa, MG, Brasil.

2 Departamento de Ciências do Esporte, Curso de Mestrado em Educação Física da Universidade Federal do Triângulo Mineiro (UFTM), Uberaba, MG, Brasil.
Deve-se ressaltar que a HAS encontra-se entre os mais importantes fatores de risco de mortalidade cardiovascular e complicações decorrentes de todo o processo como o acidente vascular cerebral (AVC) e insuficiência cardíaca congestiva ${ }^{4}$. Dentre os fatores associados à pressão arterial (PA) elevada estão os hábitos alimentares inadequados, inatividade física, consumo excessivo de álcool e o fumo ${ }^{5}$. Um recente estudo conduzido com escolares da cidade de Maceió revelou associação entre o maior status socioeconômico, Índice de Massa Corporal (IMC) e PA elevada comparados com escolares de menor renda ${ }^{6}$. Entretanto, os autores reportaram que a obesidade expli- 
cou parcialmente a PA elevada em crianças de extratos mais elevados de renda, o que sinaliza a necessidade de se explorar outros fatores, por exemplo, os níveis de atividade física (NAF).

O tempo em atividade física de moderada a vigorosa intensidade por crianças e adolescentes está associado a menores valores do perímetro de cintura, PA, níveis de insulina e triglicerídeos e maiores valores de HDL-c, independente da idade, sexo e tempo em comportamento sedentário ${ }^{7}$. Embora estudos transversais limitem inferências de causalidade, estudos de intervenção sugerem efeito do exercício físico sobre redução da PA de crianças obesas ${ }^{8}$. Araújo et $\mathrm{al}^{9} \mathrm{em}$ sua meta-análise concluíram que a prática regular de exercícios aeróbicos pode diminuir a PAS e PAD em $3 \mathrm{mmHg}$ em indivíduos normotensos, em $6 \mathrm{mmHg}$ e $7 \mathrm{mmHg}$ em indivíduos hipertensos limítrofes e em $10 \mathrm{mmHg}$ e $8 \mathrm{mmHg}$ em hipertensos graves, respectivamente. Ainda, estudo longitudinal realizado com crianças de cinco a sete anos de idade reportou que, tanto no primeiro momento, quanto dois anos após a primeira medida, a PA elevada esteve associada a baixos níveis de atividade física ${ }^{10}$.

Apesar do aumento considerado da prevalência de HAS em crianças, a aferição da PA não é prática habitual nos atendimentos e acompanhamento pediátrico. Embora existam tabelas percentílicas de referência, programas eletrônicos para facilitar a classificação e equipamentos pediátricos adequados, o subdiagnóstico em crianças está documentado na literatura ${ }^{11}$. Aprofundar o conhecimento sobre a associação de medidas rotineiramente utilizadas pela prática clínica como antropometria e outras mais específicas, mas não mais complexa, como o NAF pode ser uma alternativa para triagem de PA elevada em crianças.

Nesse contexto, alguns estudos conduzidos com crianças brasileiras em idade escolar apontaram associação entre $\mathrm{IMC}^{11-13}$, perímetro de cintura ${ }^{14}$ e PA elevada. Por outro lado, até o nosso conhecimento, não foram publicados pontos de corte de medidas antropométricas (IMC, perímetro de cintura e dobras cutâneas) e do NAF para predizer PA elevada em crianças brasileiras, por sexo, em idade escolar. Dessa forma, o objetivo do presente estudo foi verificar a acurácia das medidas antropométricas e do NAF como preditores do risco de PA elevada, por sexo, em crianças.

\section{Métodos}

Trata-se de um estudo transversal, em que participaram escolares matriculados no $5^{\circ}$ ano do ensino fundamental do município de Viçosa-MG. Segundo dados da contagem populacional do IBGE (2007), a cidade de Viçosa possuía 70.704 habitantes e destes 1.049 estavam no $5^{\circ}$ ano do ensino fundamental. Do total de escolas, 32 atendiam a faixa etária de estudo, sendo oito estaduais $(\mathrm{n}=444)$, dezoito municipais $(n=406)$ e seis privadas $(n=196)$, segundo dados da Superintendência Regional de Ensino.

Para o cálculo do tamanho mínimo amostral, utilizou-se a equação proposta por Lwanga e Lemeshow ${ }^{12}$, tendo como parâmetros o número total de escolares na faixa etária estudada, o número total da população, erro tolerável de $5 \%$ e nível de confiança de $95 \%$. A amostra mínima calculada foi de 107 crianças, porém, participaram do estudo 187 crianças, sendo 63 da rede de ensino público estadual, 64 da rede de ensino público municipal e 60 da rede particular, representada por nove escolas. Os seguintes critérios de inclusão foram atendidos: não utilização de medicamentos, não estar em dieta de restrição calórica e regularmente matriculados nas escolas sorteados do município de Viçosa-MG.

Após a aprovação do estudo pelo Comitê de Ética em Pesquisa com Seres Humanos da Universidade Federal de Viçosa (Of. Ref. $n^{\circ} 060 / 2009$ ), realizou-se 
o primeiro contato no ambiente escolar com autorização da direção das escolas. Aqueles que preencheram os critérios de inclusão e demonstraram interesse em participar do estudo, receberam o Termo de Consentimento Livre e Esclarecido para conhecimento e assinatura dos pais. Após autorização dos responsáveis, as crianças foram individualmente submetidas à avaliação antropométrica e aferição da PA, em espaço reservado disponibilizado pela escola. Em seguida, foram instruídas individualmente ao preenchimento do registro de atividades físicas e ao uso e registro do número de passos/dias do pedômetro. As avaliações foram realizadas por uma equipe de profissionais previamente treinados (enfermeiro, profissional de educação física e nutricionista).

A pressão arterial foi aferida pelo método auscultatório com esfigmomanômetro de coluna de mercúrio, com manguitos de tamanhos apropriados a circunferência dos braços das crianças, seguindo normas padronizadas ${ }^{2}$. Após repouso aproximado de 10 minutos, as crianças sentadas tiveram sua pressão aferida por três vezes com um intervalo mínimo de um minuto entre as aferições, considerou-se o valor médio das duas ultimas aferições. Só foram diagnosticadas com PA elevada as crianças que apresentaram a PA sistólica (PAS) ou PA diastólica (PAD) acima do percentil 90 (de acordo com o percentil da estatura, sexo e idade) em três dias distintos em que foram feitas as aferições ${ }^{2}$.

Para estimar o nível de atividade física (NAF) foi utilizado o registro recordatório de Bouchard et al..$^{15}$ em que se obteve a gasto energético total (GET). Para a estimativa da taxa metabólica basal (TMB) utilizou-se a fórmula FAO/WHO/UNU para crianças de 3 a 10 anos de idade. O NAF foi calculado pela divisão do GET pela TMB.

As crianças utilizaram pedômetro (Pulse Rate Pedometer, modelo DX 8897) durante sete dias consecutivos. Cada participante recebeu um impresso que continha instruções de uso e uma planilha para preencher o número de passos dados em cada dia mensurado. As instruções foram também direcionadas aos pais de forma a validar as informações do número de passos inseridas diariamente na planilha. As crianças foram recomendadas a retirar os pedômetros nos momentos de banho, atividades aquáticas e durante o sono e, posicioná-los novamente ao acordar. Para as análises, foi utilizada a média do número de passos dados nos sete dias. As recomendações foram atendidas para crianças que atingiram $11.000 \mathrm{e}$ 13.000 passos/dia para o sexo feminino e masculino, respectivamente ${ }^{16}$.

A massa corporal foi obtida por balança eletrônica digital (Plena, Lumina) com capacidade máxima de $150 \mathrm{~kg}$ e precisão de $100 \mathrm{~g}$. A estatura foi obtida com uso do antropômetro portátil (Rigor e Técnica) com comprimento de $2 \mathrm{~m}$ e escala de $0,1 \mathrm{~cm}$. O IMC foi calculado com as medidas da massa corporal e estatura por meio da fórmula: $\mathrm{IMC}=$ massa corporal $(\mathrm{kg}) /$ estatura $\left(\mathrm{m}^{2}\right)$. As crianças foram classificadas com sobrepeso e obesidade, segundo os critérios estabelecidos por Cole et al. ${ }^{13}$.

O perímetro da cintura (PC) foi obtido com uso de fita métrica flexível e inelástica com extensão de $2 \mathrm{~m}$ (Sanny) graduada em centímetros e subdividida em milímetros. As medidas foram realizadas em triplicata, e considerou-se para análise o valor médio entre elas. Foram mensurados os pontos anatômicos da menor curvatura do abdome, entre a crista-ilíaca e as costelas (PCMC), ponto médio entre a crista-ilíaca e a última costela (PCPM) e sobre a cicatriz umbilical (PCCI).

Foram obtidas as dobras cutâneas tricipital (DCT), bicipital (DCB), subescapular (DCSe) e supra-ilíaca (DCSi), utilizando-se o equipamento Lange Skinfold Caliper, que exerce pressão constante de $10 \mathrm{~g} / \mathrm{mm}^{2}$, do lado direito do corpo e com, no mínimo, três repetições não consecutivas para cada medida. A medida final 
foi obtida pela média dos três valores. Os resultados foram interpretados isoladamente, bem como pelo somatório da espessura de duas dobras (DCT+ DCSe) $(\Sigma 2 \mathrm{DC})$ e das quatro dobras cutâneas (DCT $+\mathrm{DCB}+\mathrm{DCSe}+\mathrm{DCSi})$ avaliadas $(\Sigma$ 4DC), com os resultados expressos em milímetros ( $\mathrm{mm}$ ).

Para o cálculo do percentual de gordura corporal (\% GC), foram utilizadas as seguintes equações propostas por Slaughter et $\mathrm{al}^{17}$.

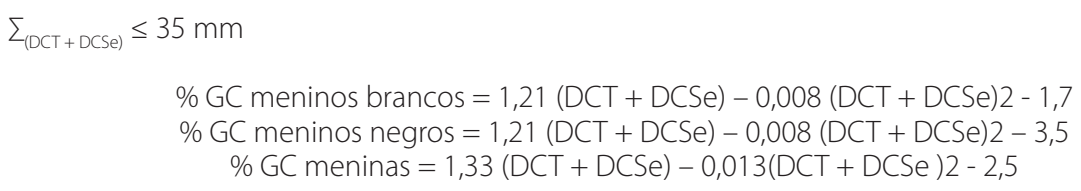

Foram utilizados para as análises estatísticas os softwares SPSS 17.0 e Medcalc 11.0.1. Todas as variáveis foram testadas quanto a sua normalidade pelo teste de Kolmogorov-Smirnov. O teste $t$ de Student foi utilizado para comparação entre grupos independentes com distribuição normal e o teste de Mann-Whitney para comparação entre grupos com distribuição não paramétrica.

Curvas Receiver Operating Characteristic (ROC) foram utilizadas para determinar pontos de corte de medidas antropométricas e NAF, com melhor equilíbrio entre sensibilidade e especificidade, em predizer níveis pressóricos elevados em crianças. A significância estatística de cada análise foi verificada pela área sob a curva (AUC) ROC e pelo limite inferior do intervalo de confiança (IC) a 95\% maior que 0,5.

\section{Resultados}

Participaram do estudo 187 crianças (74,8\% das crianças convidadas), com idade média de 9,9 $\pm 0,7$ anos, das quais $56,7 \%$ eram do sexo feminino. As taxas de resposta do registro recordatório de Bouchard ${ }^{15}$ e de utilização do pedômetro durante os sete dias foram de $62 \%$ e $72,7 \%$, respectivamente.

PAS e PAD não diferiram ( $p>0,05)$ entre os sexos (Tabela 1) e a prevalência de PA elevada foi $14,4 \%$ e $13,6 \%$ entre meninos e meninas, respectivamente.

TABELA 1 - Pressão arterial, estratificado por sexo, escolares do $5^{\circ}$ ano do ensino fundamental do município de Viçosa-MG, 2009.

\begin{tabular}{lcccc}
\hline & \multicolumn{2}{c}{ Meninas $(\mathrm{n}=106)$} & \multicolumn{2}{c}{ Meninos $(\mathrm{n}=81)$} \\
\cline { 2 - 5 } & Média $\pm \mathrm{dp}$ & Mediana (min-max) & Média $\pm \mathrm{dp}$ & Mediana (min - max $)$ \\
\hline PAS $(\mathrm{mmHg})$ & $110,2 \pm 10,2$ & $110(90-140)$ & $107,0 \pm 9,4$ & $108(90-140)$ \\
\hline PAD $(\mathrm{mmHg})$ & $73,5 \pm 7,5$ & $70(60-100)$ & $72,2 \pm 7,5$ & $70(56-100)$ \\
\hline
\end{tabular}

Legenda: PAS = pressão arterial sistólica; PAD = pressão arterial diastólica.

O número de passos por dia e o NAF (Tabela 2) não diferiu significativamente entre os sexos ( $p>0,05)$. A recomendação de 11.000 e 13.000 passos/dia foi atingida por $13,6 \%$ das meninas e $14,5 \%$ dos meninos, respectivamente.

Massa corporal, estatura, IMC e perímetro de cintura não diferiram significativamente entre os sexos $(p>0,05)$. Por outro lado, meninas apresentaram valores significativamente superiores em relação aos meninos $(\mathrm{p}<0,05)$ para todas as dobras cutâneas, $\Sigma$ 2DC, $\Sigma$ 4DC e para \%GC (Tabela 3). 
A prevalência de crianças com sobrepeso foi de $15 \%$ e obesidade $5,9 \%$. Os meninos apresentaram maior prevalência de obesidade em relação às meninas $(8,6 \%$ vs. 3,8\%, $\mathrm{p}<0,05)$.

TABELA 2 - Número de passos e nível de atividade física, por sexo, escolares do $5^{\circ}$ ano do ensino fundamental do município de Viçosa-MG, 2009.

\begin{tabular}{lcccc}
\hline & \multicolumn{2}{c}{ Meninas } & \multicolumn{2}{c}{ Meninos } \\
\cline { 2 - 5 } & Média $\pm d p$ & Mediana (min- max) & Média $\pm d p$ & Mediana (min- max) \\
\hline Passos/dia $(n=136)$ & $5.982 \pm 4.740$ & $4.906(387-20.803)$ & $7.468 \pm 6.005$ & $5.952(807-31.459)$ \\
\hline NAF $(n=116)$ & $2,9 \pm 0,9$ & $2,8(1,7-7,3)$ & $3,1 \pm 1,0$ & $2,9(1,3-5,3)$ \\
\hline
\end{tabular}

$\mathrm{NAF}=$ nível de atividade física.

TABELA 3 - Antropometria e composição corporal, por sexo, escolares do $5^{\circ}$ ano do ensino fundamental do município de Viçosa-MG, 2009.

\begin{tabular}{|c|c|c|c|c|}
\hline \multirow[b]{2}{*}{ Variáveis } & \multicolumn{2}{|c|}{ Meninas $(n=106)$} & \multicolumn{2}{|c|}{ Meninos $(n=81)$} \\
\hline & Média $\pm d p$ & Mediana (min- max) & Média $\pm d p$ & Mediana (min- max) \\
\hline Massa corporal (kg) & $35,0 \pm 8,1$ & $33,0(23,2-59,5)$ & $35, \pm 9,4$ & $32,4(22,6-60,1)$ \\
\hline Estatura (m) & $1,4 \pm 0,7$ & $1,4(1,3-1,6)$ & $1,4 \pm 0,7$ & $1,4(13-1,6)$ \\
\hline IMC $\left(\mathrm{kg} / \mathrm{m}^{2}\right)$ & $17,6 \pm 2,9$ & $16,9(13,7-29,1)$ & $17,7 \pm 3,6$ & $16,5(13,3-28,7)$ \\
\hline PCPM (cm) & $62,1 \pm 8,3$ & $59,4(50,4-93,5)$ & $62,2 \pm 9,2$ & $59,7(52,0-93,2)$ \\
\hline $\mathrm{PCCl}(\mathrm{cm})$ & $65,2 \pm 8,3$ & $62,5(53,2-90,7)$ & $64,3 \pm 10,4$ & $61,2(44,2-94,9)$ \\
\hline PCMC (cm) & $59,8 \pm 6,8$ & $57,7(51,0-82,5)$ & $61,3 \pm 8,5$ & $59,0(51,3-88,0)$ \\
\hline $\mathrm{DCT}(\mathrm{mm})^{*}$ & $16,7 \pm 7,2$ & $14,7(4,8-38,7)$ & $14,4 \pm 8,4$ & $12,0(4,0-41,3)$ \\
\hline $\mathrm{DCB}(\mathrm{mm})^{*}$ & $9,5 \pm 4,9$ & $7,3(3,0-22,0)$ & $8,2 \pm 6,0$ & $6,0(3,0-31,3)$ \\
\hline DCSi $(m m)^{*}$ & $14,9 \pm 11,0$ & $10,5(4,0-52,5)$ & $11,8 \pm 11,0$ & $7,5(3,0-59,0)$ \\
\hline DCSe $(\mathrm{mm})^{*}$ & $11,5 \pm 7,8$ & $8,0(4,0-35,3)$ & $10,3 \pm 8,6$ & $7,0(4,0-40,0)$ \\
\hline$\sum 2 \mathrm{DC}(\mathrm{mm})^{*}$ & $28,2 \pm 13,9$ & $28,2(9,8-67,3)$ & $24,7 \pm 16,5$ & $18,7(8,0-76,3)$ \\
\hline$\sum 4 \mathrm{DC}(\mathrm{mm})^{*}$ & $52,7 \pm 28,5$ & $41,9(18,7-140,3)$ & $44,7 \pm 32,8$ & $32,0(14,0-166,7)$ \\
\hline$\% \mathrm{GC}^{*}$ & $22,2 \pm 6,1$ & $21,1(9,3-31,5)$ & $18,9 \pm 6,6$ & $17,8(7,3-31,3)$ \\
\hline
\end{tabular}

IMC = índice de massa corporal; PCPM = perímetro de cintura ponto médio entre crista ilíaca e a última costela; $\mathrm{PCCl}=$ perímetro de cintura sobre a cicatriz umbilical; $\mathrm{PCMC}=$ perímetro de cintura na menor curvatura; DCT = dobra cutânea triciptal; DCB = dobra cutânea biciptal; DCSi = dobra cutânea supra-ilíaca; DCSe = dobra cutânea subescapular; $\Sigma 2$ DC = soma das dobras cutâneas triciptal e subescapular; $\Sigma 4 D C=$ soma das quatro dobras cutâneas avaliadas; \% GC = percentual de gordura corporal. *Diferença significativa entre os sexos $p<0,05$ teste de Mann Whitney.

IMC, PCPM, PCCI, PCMC, $\Sigma$ 4DC e NAF foram acurados em predizer PA elevada entre meninas (Tabela 4). Para os meninos, apenas o número de passos $(<6.640$ por dia) foi acurado em predizer PA elevada, com AUC = 0,67 [(IC 95\%= 0,53-0,80), $p=0,02]$, sensibilidade de $62,5 \%$ e especificidade de $65,1 \%$.

TABELA 4 - Variáveis preditoras da pressão arterial elevada em escolares do $5^{\circ}$ ano do ensino fundamental do sexo feminino do município de Viçosa/MG, 2009.

\begin{tabular}{lcccccc}
\hline Variáveis preditoras & AUC & $p$ & $I C 95 \%$ & $P C$ & SE & ES \\
\hline IMC & 0,7 & 0,04 & $0,5-0,9$ & $>18,1$ & 68,7 & 73,3 \\
\hline PCPM & 0,7 & 0,02 & $0,5-0,9$ & $>61,0$ & 75,0 & 62,0 \\
\hline PCCI & 0,7 & 0,03 & $0,6-0,8$ & $>63,0$ & 68,7 & 63,3 \\
\hline PCMC & 0,7 & 0,04 & $0,6-0,8$ & $>59,0$ & 68,7 & 64,4 \\
\hline$\sum 4$ DC & 0,7 & 0,04 & $0,5-0,8$ & $>40,7$ & 75,0 & 61,0 \\
\hline NAF & 0,7 & 0,01 & $0,6-0,9$ & $<2,8$ & 75,0 & 67,1 \\
\hline
\end{tabular}

$\mathrm{AUC}$ = área sob a curva $\mathrm{ROC} ; \mathrm{PC}=$ ponto de corte; $\mathrm{SE}=$ sensibilidade; $\mathrm{ES}=$ especificidade; $I \mathrm{MC}=$ índice de massa corporal; PCPM = perímetro de cintura ponto médio entre crista ilíaca e a última costela; $\mathrm{PCCl}=$ perímetro de cintura sobre a cicatriz umbilical; $\mathrm{PCMC}=$ perímetro de cintura na menor curvatura; $\Sigma 4 D C$ = soma das quatro dobras cutâneas avaliadas; NAF = nível de atividade física. 


\section{Discussão}

O presente estudo investigou acurácia de medidas antropométricas e NAF em predizer PA elevada em crianças. Apresentamos pontos de corte de IMC, perímetros de cintura, $\Sigma$ 4DC e NAF para meninas e do número de passos/dia para meninos como preditores de PA elevada, acompanhados dos respectivos valores de sensibilidade e especificidade. Nossos achados se traduzem numa proposta simples, de fácil aplicabilidade e custo reduzido para ser utilizada pelas escolas, em parceria com as secretarias de saúde dos municípios, pois constitui meta a ser alcançada no âmbito dos Ministérios da Educação e da Saúde por meio do Programa Saúde na Escola.

A prevalência de PA elevada no presente estudo foi de 14\%, sendo 13,6\% nas meninas e $15,1 \%$ nos meninos. Estes achados corroboram com a prevalência encontrada de PA elevada $13,6 \%{ }^{4}$ em crianças brasileiras. Por outro lado, Moura et al. ${ }^{18}$ apresentaram menores valores de prevalência de PA elevada em crianças $(6,5 \%)$ e adolescentes $(7,7 \%)$ brasileiros. Dados de prevalência contribuem para nortear intervenções e políticas públicas com objetivo de controlar o fenômeno em estudo. Assim, os resultados apresentados sinalizam para necessidade de mais estudos haja vista associação entre PA elevada na infância e HAS na idade adulta ${ }^{19}$.

No presente estudo, o ponto de corte do PCPM para predizer PA elevada foi $>60 \mathrm{~cm}$. Ferreira et $\mathrm{al}^{20}$ identificaram em crianças, o perímetro da cintura acima de $78 \mathrm{~cm}$ como preditor da síndrome metabólica, caracterizada como conjunto de alterações em pelo menos três dos cinco componentes metabólicos (HDL-c, glicemia, triglicerídeos, perímetro de cintura e PA). Estudos de predição da PA em crianças são escassos. Em adolescentes, Beck et al. ${ }^{21}$ corroboraram com nossos achados, e encontraram perímetro da cintura como a melhor medida antropométrica na predição de PA elevada.

As medidas antropométricas apresentam-se como parâmetro importante na detecção de PA elevada, principalmente o perímetro de cintura. Estudo com 2334 escolares de sete anos de idade de Taiwan ${ }^{22}$ reportou que crianças classificadas no quarto quartil da razão cintura-estatura apresentaram maior prevalência de PA elevada $(31,2 \%)$ quando comparadas aquelas no primeiro quartil $(8,8 \%)$. Os valores médios da razão cintura-estatura foram superiores nas crianças com PA elevada quando comparadas aquelas que apresentaram valores normais de PA.

O perímetro de cintura, enquanto medida de obesidade central, associa-se especificamente aos fatores de risco das doenças cardiovasculares nos adultos e crianças, o que o torna medida relevante para avaliar risco de doenças crônicas como a $\mathrm{HAS}^{4}$. A importância de estabelecer pontos de corte para medidas antropométricas de adiposidade central é reforçada devido ao fato de que a obesidade central apresenta forte associação com a ocorrência de infarto agudo do miocárdio ${ }^{23} \mathrm{e}$ pode ser o melhor indicador para o controle da PA em populações pediátricas ${ }^{21}$.

Levando em consideração a importância da prática regular de atividades físicas para a saúde, os achados do presente estudo indicam baixa frequência (14\%) de crianças que atendem as recomendações mínimas para serem consideradas fisicamente ativas ${ }^{16}$. Dados sobre o número de passos em crianças, avaliados pelo pedômetro, ainda são escassos na literatura, porém ao avaliar crianças e adolescentes canadenses, na faixa etária de 5 a 19 anos, Tudor-Locke et al ${ }^{24}$ identificaram média de 12.813 passos/dia na faixa etária de cinco a nove anos e de 12.845 passos/dia na faixa etária de 10 a 13 anos. Ao avaliar a atividade física habitual obtido pelo pedômetro em 162 indivíduos de 10 a 14 anos de idade, Rosa et $\mathrm{al}^{25}$ observaram que meninos e meninas com idades inferiores a 14 anos obtiveram média de 12.514 passos/dia e de 9.502 passos/dia, respectivamente. 
Nota-se entre os estudos que utilizaram o pedômetro para avaliar a prática de atividade física habitual que os meninos executam maior número de passos/dia quando comparados as meninas. Este fato é explicado por Goncalves et $\mathrm{al}^{26}$ quando destacam que para os meninos é dado maior incentivo familiar para a prática de atividade física, o que os levam a praticar maior quantidade de atividades físicas vigorosas.

Por se tratar de medida objetiva, o pedômetro se apresenta como instrumento mais preciso para avaliação do NAF quando comparado ao recordatório de atividades e outros questionários ${ }^{27}$. Apesar de apresentar limitações, principalmente quando utilizado em crianças $^{28}$, acredita-se que a medida obtida pelo pedômetro possa reportar valores mais próximos da atividade física habitual de crianças quando comparados aos recordatórios e questionários ${ }^{27}$.

Além da interferência positiva da prática regular de atividade física no controle da PA, encontra-se na literatura indicações de que esse hábito também exerce influência no controle e tratamento do excesso de peso em crianças e adolescentes. Por exemplo, crianças e adolescentes que não cumpriram as recomendações do NAF, avaliados por pedômetro, apresentaram valores superiores de adiposidade corporal, avaliados pelo IMC e pela bioimpedância, quando comparados aos que cumpriram as recomendações de números de passos/dia ${ }^{25}$.

Estudos demonstram que a obesidade é um importante fator de risco para PA elevada. A chance de crianças obesas apresentarem PA elevada foi aproximadamente 4 vezes superior do que a observada em não obesos ${ }^{29}$. Costanzi et al ${ }^{30}$ observaram que crianças com perímetro de cintura aumentado apresentaram 2,8 vezes mais chance de ter PA elevada do que crianças com perímetro de cintura adequado e que o percentual de crianças com PA elevada foi estatisticamente maior $(\mathrm{p}=0,001)$ nas crianças consideradas obesas ou com sobrepeso.

No presente estudo, o IMC apresentou boa área sob a curva ROC, porém não foi a variável melhor preditora do risco de PA elevada, como apresentado por de Rosa et $\mathrm{al}^{23}$. No estudo de Ferreira et $\mathrm{al}^{20}$ o IMC acima de $24,5 \mathrm{Kg} / \mathrm{m}^{2}$ e o \%GC acima de $41 \%$, além do perímetro da cintura, foram preditores da síndrome metabólica em crianças.

O presente estudo apresenta algumas limitações que devem ser levadas em consideração no momento de interpretação e uso dos resultados, por exemplo: 1) utilização do recordatório para mensuração do NAF está sujeito ao viés de memória por parte das crianças; 2) embora o estudo envolvesse apenas crianças do $5^{\circ}$ ano do ensino fundamental, os resultados aqui apresentados devem ser confirmados por futuros estudos para outras faixas etárias.

Concluiu-se que IMC, perímetro de cintura medido em três pontos anatômicos distintos, $\Sigma 4$ DC e NAF para meninas e o número de passos/dia para os meninos foram acurados em predizer PA elevada. A prática regular da atividade física e o controle do perímetro de cintura são determinantes para saúde cardiovascular e são ferramentas úteis e de fácil manuseio para consolidação de políticas públicas de prevenção e controle da HAS.

\section{Agradecimentos}

Os autores agradecem aos diretores das escolas pela colaboração e disponibilização dos espaços para realização das coletas, aos pais e responsáveis pelas autorizações e as crianças pela disponibilidade de participação. 


\section{Contribuiç̧ão dos autores}

Alynne C. R. Andaki participou do planejamento, coleta e análise dos dados e redação do manuscrito. Edmar L. Mendes contribuiu na coleta, análise dos dados e correção do texto. Fernanda S. Franco e Wellington Segheto participaram da redação das sessões introdução e discussão. Adelson L. A. Tinôco orientou os procedimentos para coletas e revisou o texto.

\section{Referências}

1. Dong B, Wang Z, Song Y, Wang HJ, Ma J. Understanding trends in blood pressure and their associations with body mass index in Chinese children, from 1985 to 2010: a crosssectional observational study. BMJ open. 2015;5(9):e009050.

2. Sociedade Brasileira de C, Sociedade Brasileira de H, Sociedade Brasileira de N. [VI Brazilian Guidelines on Hypertension]. Arq Bras Cardiol. 2010;95(1 Suppl):1-51.

3. Magalhaes MG, Oliveira LM, Christofaro DG, Ritti-Dias RM. Prevalence of high blood pressure in Brazilian adolescents and quality of the employed methodological procedures: systematic review. Rev Bras Epidemiol. 2013;16(4):849-59.

4. Queiroz VM, Moreira PV, Vasconcelos TH, Toledo Vianna RP. Prevalence and anthropometric predictors of high blood pressure in schoolchildren from Joao Pessoa PB, Brazil. Arq Bras Cardiol. 2010;95(5):629-34.

5. Salgado CM, Carvalhaes JT. [Arterial hypertension in childhood]. J Pediatr (Rio J). 2003;79 Suppl 1:S115-24.

6. Ferreira HS, Lucio GM, Assuncao ML, Silva BC, Oliveira JS, Florencio TM, et al. High Blood Pressure among Students in Public and Private Schools in Maceio, Brazil. PloS one. $2015 ; 10(11): \mathrm{e} 0142982$.

7. Ekelund U, Luan J, Sherar LB, Esliger DW, Griew P, Cooper A. Moderate to vigorous physical activity and sedentary time and cardiometabolic risk factors in children and adolescents. Jama. 2012;307(7):704-12.

8. Garcia-Hermoso A, Saavedra JM, Escalante Y. Effects of exercise on resting blood pressure in obese children: a meta-analysis of randomized controlled trials. Obesity reviews: an official journal of the International Association for the Study of Obesity. 2013;14(11):919-28.

9. Araujo TL, de Lopes MV, Cavalcante TF, Guedes NG, Moreira RP, Chaves ES, et al. [Analysis of risk indicators for the arterial hypertension in children and teenagers]. Rev Esc Enferm USP. 2008;42(1):120-6.

10. Knowles G, Pallan M, Thomas GN, Ekelund U, Cheng KK, Barrett T, et al. Physical activity and blood pressure in primary school children: a longitudinal study. Hypertension. 2013;61(1):70-5.

11. Fuly JT, Giovaninni NP, Marcato DG, Alves ER, Sampaio JD, Moraes LI, et al. Evidence of underdiagnosis and markers of high blood pressure risk in children aged 6 to 13 years. J Pediatr (Rio J). 2014;90(1):65-70.

12. Moraes LI, Nicola TC, Jesus JS, Alves ER, Giovaninni NP, Marcato DG, et al. High blood pressure in children and its correlation with three definitions of obesity in childhood. Arq Bras Cardiol. 2014;102(2):175-80.

13. Rosaneli CF, Baena CP, Auler F, Nakashima AT, Netto-Oliveira ER, Oliveira AB, et al. Elevated blood pressure and obesity in childhood: a cross-sectional evaluation of 4,609 schoolchildren. Arq Bras Cardiol. 2014;103(3):238-44.

14. Burgos MS, Burgos LT, Camargo MD, Franke SIR, Prá D, da Silva AMV, et al. Relationship between Anthropometric Measures and Cardiovascular Risk Factors in Children and Adolescents. Arq Bras Cardiol. 2013;101(4):288-96.

15. Bouchard C, Tremblay A, Leblanc C, Lortie G, Savard R, Theriault G. A method to assess energy expenditure in children and adults. Am J Clin Nutr. 1983;37(3):461-7.

16. Tudor-Locke C, Craig CL, Beets MW, Belton S, Cardon GM, Duncan S, et al. How many steps/day are enough? for children and adolescents. Int J Behav Nutr Phys Act. 2011;8:78.

17. Slaughter MH, Lohman TG, Boileau RA, Horswill CA, Stillman RJ, Van Loan MD, et al. Skinfold equations for estimation of body fatness in children and youth. Hum Biol. 1988;60(5):709-23.

18. Moura AA, Silva MA, Ferraz MR, Rivera IR. [Prevalence of high blood pressure in children and adolescents from the city of Maceio, Brazil]. J Pediatr (Rio J). 2004;80(1):35-40. 
19. Bassareo PP, Mercuro G. Pediatric hypertension: An update on a burning problem. World J Cardiol. 2014;6(5):253-9.

20. Ferreira AP, Ferreira CB, Brito CJ, Pitanga FJ, Moraes CF, Naves LA, et al. Prediction of metabolic syndrome in children through anthropometric indicators. Arq Bras Cardiol. 2011;96(2):121-5.

21. Beck CC, Lopes Ada S, Pitanga FJ. Anthropometric indicators as predictors of high blood pressure in adolescents. Arq Bras Cardiol. 2011;96(2):126-33.

22. Chen TL, Choy CS, Chan WY, Chen CH, Liao CC. Waist to height ratio and elevated blood pressure among children in Taiwan. Indian Pediatr. 2012;49(6):463-6.

23. Rosa ML, Mesquita ET, da Rocha ER, Fonseca Vde M. Body mass index and waist circumference as markers of arterial hypertension in adolescents. Arq Bras Cardiol. 2007;88(5):573-8.

24. Tudor-Locke C, Craig CL, Cameron C, Griffiths JM. Canadian children's and youth's pedometer-determined steps/day, parent-reported TV watching time, and overweight/ obesity: the CANPLAY Surveillance Study. Int J Behav Nutr Phys Act. 2011;8:66.

25. Rosa CSdC, Messias KP, Fernandes RA, Silva CBd, Monteiro HL, Freitas Júnior IF. Atividade física habitual de crianças e adolescentes mensurada por pedômetro e sua relação com índices nutricionais. Rev Bras Cineantropom Desempenho Hum. (Online). 2011:22-8.

26. Goncalves H, Hallal PC, Amorim TC, Araujo CL, Menezes AM. [Sociocultural factors and physical activity level in early adolescence]. Rev Panam Salud Publica. 2007;22(4):246-53.

27. Harris TJ, Owen CG, Victor CR, Adams R, Ekelund U, Cook DG. A comparison of questionnaire, accelerometer, and pedometer: measures in older people. Med Sci Sports Exerc. 2009;41(7):1392-402.

28. Eisenmann JC, Wickel EE. Moving on land: an explanation of pedometer counts in children. Eur J Appl Physiol. 2005;93(4):440-6.

29. Nogueira PC, da Costa RF, Cunha JS, Silvestrini L, Fisberg M. [High arterial pressure in school children in Santos--relationship to obesity]. Rev Assoc Med Bras. 2007;53(5):426-32.

30. Costanzi CB, Halpern R, Rech RR, Bergmann ML, Alli LR, Mattos AP. Associated factors in high blood pressure among schoolchildren in a middle size city, southern Brazil. J Pediatr (Rio J). 2009;85(4):335-40.

\section{ENDEREÇO PARA}

CORRESPONDÊNCIA

ALYNNE CHRISTIAN RIBEIRO ANDAKI

alynneandaki@yahoo.com.br
Universidade Federal do Triângulo

Mineiro - UFTM. Departamento de

Ciências do Esporte - DCEs.

Av. Getúlio Guaritá, n. 159 - Centro

Educacional, Sala 313-E, Bairro: Na

Sra. da Abadia - CEP: 38025-440 -

UBERABA/MG, Fone: (34) 3318-5964
RECEBIDO

REVISADO

$27 / 10 / 2015$

$22 / 12 / 2015$

APROVADO 29/01/2016 